Document downloaded from:

http://hdl.handle.net/10251/55416

This paper must be cited as:

Soto Pacheco, P.; Boria Esbert, VE.; Carceller Candau, C.; Vicente Quiles, CP.; Gil Raga, J.; Gimeno Martinez, B. (2012). EM Based Synthesis and Design of Bandpass Waveguide Filters Including Manufacturing Effects with FEST3D. International Journal of RF and Microwave Computer-Aided Engineering. 22(1):93-103. doi:10.1002/mmce.20588.

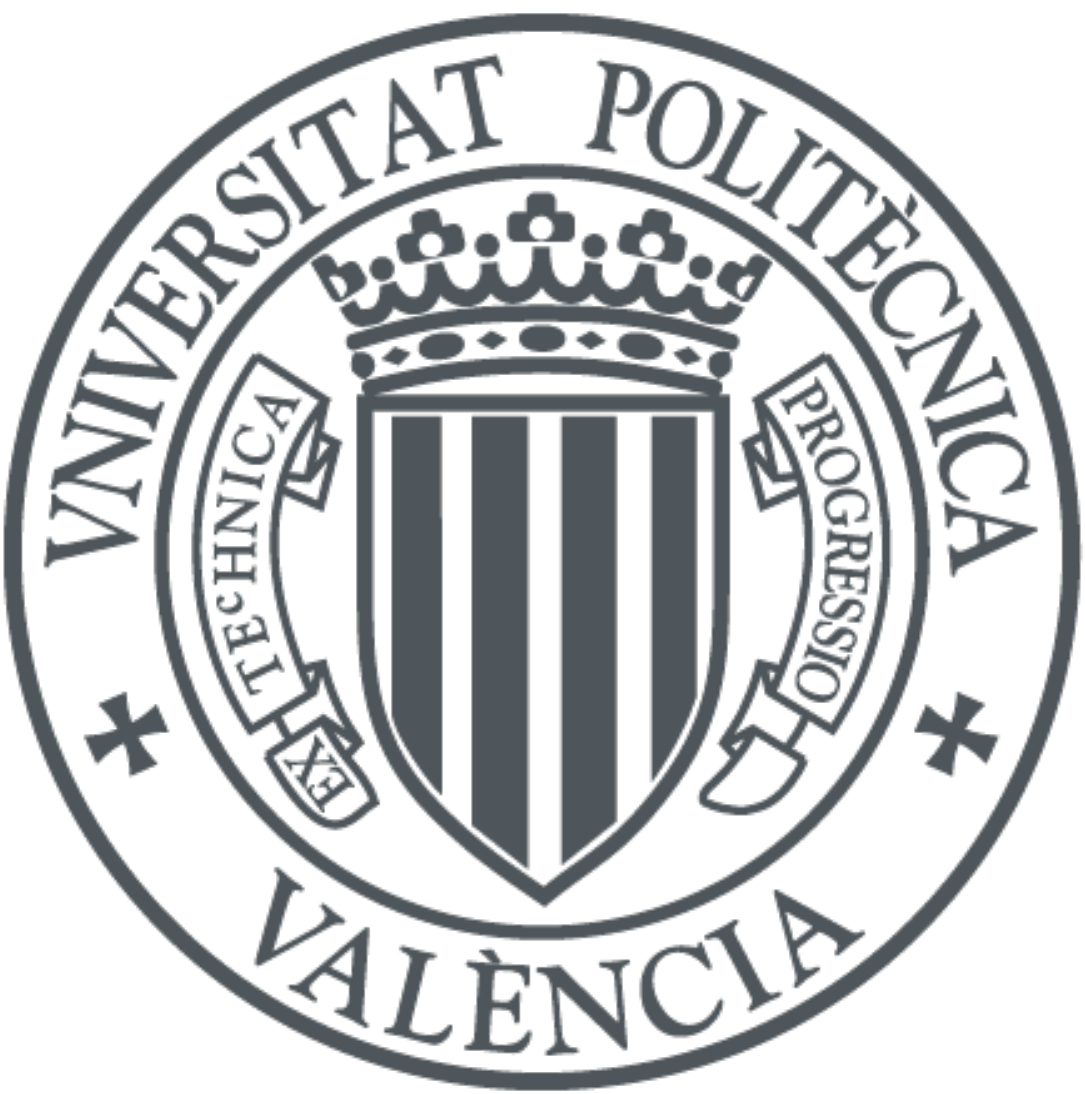

The final publication is available at

http://dx.doi.org/10.1002/mmce.20588

Copyright Wiley

Additional Information 


\title{
EM-BASED SYNTHESIS AND DESIGN OF BANDPASS WAVEGUIDE FILTERS INCLUDING MANUFACTURING EFFECTS WITH FEST3D
}

\author{
Pablo Soto*, Vicente E. Boria ${ }^{*}$, Carlos Carceller ${ }^{*}$, Carlos P. Vicente ${ }^{\dagger}$, Jordi Gil ${ }^{\dagger}$ and Benito Gimeno ${ }^{\#}$ \\ * Grupo de Aplicaciones de las Microondas - iTEAM, Universitat Politècnica de Valencia, Camino de Vera s/n, E-46022 \\ Valencia, Spain \\ ${ }^{\dagger}$ Aurora Software and Testing S.L., Edificio de Desarrollo Empresarial 9B, Camino de Vera s/n, E-46022 Valencia, Spain \\ \# Departamento de Física Aplicada - ICMUV, Universitat de València, Dr. Moliner 50, E-46100 Burjasot, Valencia, Spain
}

\begin{abstract}
This paper aims at the industry interest on automated design tools able to take into account manufacturing effects. Firstly, an efficient design strategy for bandpass waveguide filters including the rounded corners arising from low-cost manufacturing procedures is presented. This technique is based on a recent enhanced prototype and synthesis methodology able to consider the real structure parts. Using the resulting EM-based synthesis technique, an excellent structure is extracted which requires, at most, only a slight final EM optimization. Secondly, this paper presents analytical expressions providing error estimates for the different filter performances in terms of manufacturing tolerances. From such expressions, designers can determine the tolerance to be requested for a tuning-less implementation. Moreover, they can also be used to set the convergence criterion for the synthesis procedure. A fully automated design tool of bandpass waveguide filters able to consider manufacturing impairments has been developed and integrated in the commercial EM software tool FEST3D.
\end{abstract}

Keywords: Computer Aided Design, Microwave Filters, Bandpass Filters, Manufacturing, Tolerance Analysis, Rounded Corners

\section{INTRODUCTION}

Manufacturing procedures of waveguide components usually produce small variations in the structure geometry and slight random deviations in its physical dimensions. These unwanted but unavoidable effects degrade the measured response of real devices. Probably the most common geometrical modification is the conversion of sharp corners into rounded ones due to milling machining processes. The radii of such rounded corners alter the passband bandwidth, center frequency and return loss [1]. Tuning elements have been traditionally used to compensate for these impairments and the ones related to manufacturing tolerances [2]. The use of tuning elements, however, increments the component development times, costs, and undesired high-power effects, and should be avoided if possible. In fact, microwave industry is interested in lowcost tuning-less structure implementations.

In the last two decades, the increasing capabilities of modern computers have boosted an outstanding advancement on full-wave electromagnetic (EM) analysis techniques [3]-[5]. Since EM analysis tools are able to consider arbitrarily shaped geometries nowadays, several techniques have been proposed to design tuning-less waveguide filters including rounded corners [6]-[9]. Unfortunately, the rigorous analysis of structures with rounded corners is expensive in terms of CPU time, and most of these design techniques make an exhaustive use of EM simulations of the entire structure. As a result, the time required to complete the design is high, even for low and moderate order filters and after using modern space mapping approaches [6],[9].

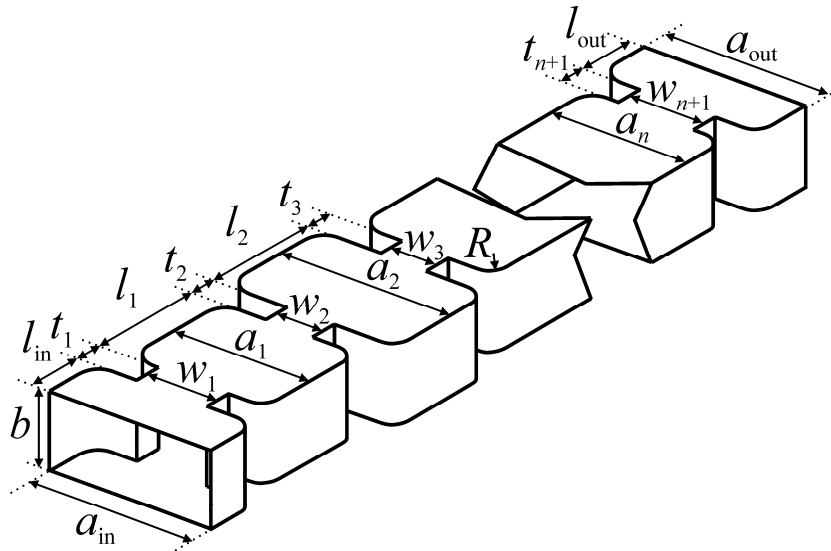

Figure 1. H-plane rectangular waveguide filter with rounded corners, and significant physical dimensions.

An important efficiency improvement can be achieved by introducing the rounded corners in the dimensional synthesis step. Traditional synthesis techniques, however, are based on analytically synthesizable prototypes which cannot precisely model the behavior of the structure elements [10],[11]. Prototypes and synthesis techniques able to include accurate information of the real filter parts obtained from full-wave EM simulations will yield better extracted structures.

A novel design methodology has been recently proposed [12],[13]. This methodology is based on a very general and flexible prototype able to acquire the wideband behavior of the real structure parts. The prototype similarity to the real structure is favored at the expense of an elaborated synthesis procedure, which is no longer analytic. An iterative technique which continuously aligns the prototype and the 


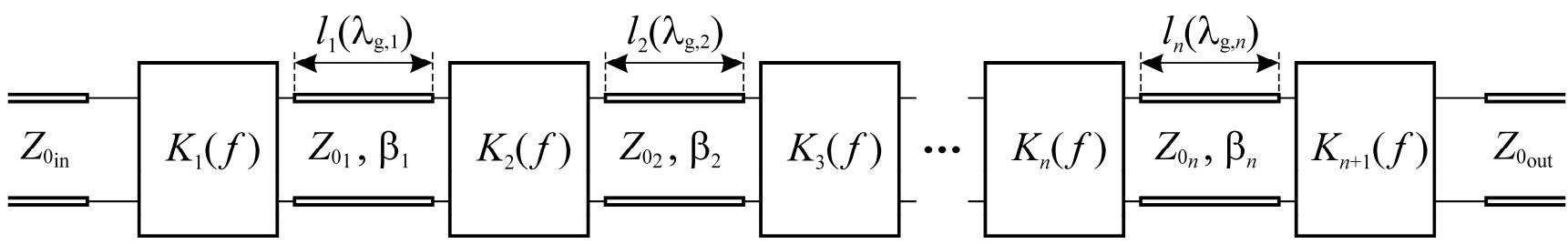

Figure 2. Prototype used to model the wideband behaviour of H-plane inductive waveguide filters with rounded corners.

real structure is obtained. This synthesis procedure extracts excellent structure dimensions, thus dramatically reducing the design time. In most cases, the final optimization step is not even required. Similar joint prototype-structure synthesis procedures were proposed in [14], [15] for the design of side-coupled coaxial cavity filters without resorting to bruteforce EM optimizations. However, these methods were conceived for narrowband filters with tuning elements, which allows performing a final adjustment of the structure response.

On the other hand, there is a lack of studies on the impact of manufacturing tolerances in structure performance. To evaluate this impact, a Monte Carlo tolerance analysis on the designed filter is usually performed. This procedure is cumbersome from a CPU point of view, especially if different tolerances must be attempted to estimate the device sensitivity and/or complex geometries are involved. To the authors' best knowledge, only the work reported in [16] has faced up to this problem by defining a criticality ratio and criticality constant to evaluate H-plane filter sensitivities. This valuable and practical contribution can be considered as a preliminary work, since both criticality terms were set based on intuition and were tested only on a limited number of filters. Moreover, the computed sensitivity always corresponds to a fixed $20 \%$ deterioration in the passband return loss.

This paper addresses the industry interest on automated design tools able to consider manufacturing impairments. Following [13], an efficient and fully automated EM-based synthesis and design technique of H-plane waveguide filters with rounded corners (see Fig. 1) has been developed. This paper also presents accurate estimators for the degradation in the filter performances due to manufacturing tolerances. From the filter specifications, the designer can determine the manufacturing tolerance required to perform a tuning-less filter implementation. Due to its practical application, these advances has been integrated within the ESA (European Space Agency) software package FEST 3D (Full-Wave Electromagnetic Simulation Tool 3D) [17].

\section{SYNTHESIS AND DESIGN PROCEDURE}

\section{A. Synthesis Methodology}

The design of H-plane waveguide filters with rounded corners has been carried out by using the synthesis methodology described in [13]. This subsection includes a brief description of this methodology for completeness.
To accurately represent the wideband behavior of the structures under consideration, the prototype proposed in [13] has been chosen (see Fig. 2). A monomode prototype has been used since the higher-order mode effects between adjacent coupling windows are usually insignificant in these particular structures. The prototype has frequency-dependant inverters with unnormalized parameter $K$ given by

$$
K_{i}(f)=K_{0, i}\left(\frac{f}{f_{0, \text { inv }}}\right)^{\alpha_{i}}
$$

and the prototype transmission line lengths also depend on frequency according to

$$
l_{i}\left(\lambda_{\mathrm{g}, i}\right)=\frac{\lambda_{\mathrm{g} 0, i}}{2}+\kappa_{\mathrm{l}, i}\left(\frac{1}{\lambda_{\mathrm{g}, i}}-\frac{1}{\lambda_{\mathrm{g} 0, i}}\right)+\kappa_{2, i}\left(\frac{1}{\lambda_{\mathrm{g}, i}}-\frac{1}{\lambda_{\mathrm{g} 0, i}}\right)^{2}
$$

where $K_{0, i}$ is the unnormalized impedance parameter of the $i$-th inverter at $f_{0, \text { inv }}$ (an arbitrary reference frequency normally set to the geometrical mean of the passband cutoff frequencies), and $\lambda_{\mathrm{g} 0, i}$ is the guide wavelength $\lambda_{\mathrm{g}, i}$ of the $i$ th structure resonator at its resonant frequency $f_{0, i}$.

The free parameters $\alpha_{i}, \kappa_{1, i}$ and $\kappa_{2, i}$ in (1)-(2) are used to model the behavior of the real filter elements in the filter passband. They are obtained from least-square fits in the form of (1) - (2) to the frequency variation of (i) the parameter $K$ provided by the real coupling window and (ii) their input/output correction lengths required to obtain the $90^{\circ}$ phase shift of an ideal inverter. These least-square fits are carried out in a wide frequency range encompassing the filter passband.

The combined prototype-structure synthesis procedure begins with the synthesis of the prototype for a suitable initial choice of its free parameters. Next, the dimensions of the structure parts implementing the different prototype elements are obtained. The wideband responses of the extracted coupling windows are then computed to update the prototype free parameters, and a new iteration starts with the synthesis of the resulting prototype. This iterative procedure stops once the difference in the physical dimensions of two consecutive extracted structures is lower than the convergence bound. The convergence criterion will be discussed in subsection III-B. For an in-depth description of the synthesis procedure, the reader is referred to [12], [13]. 


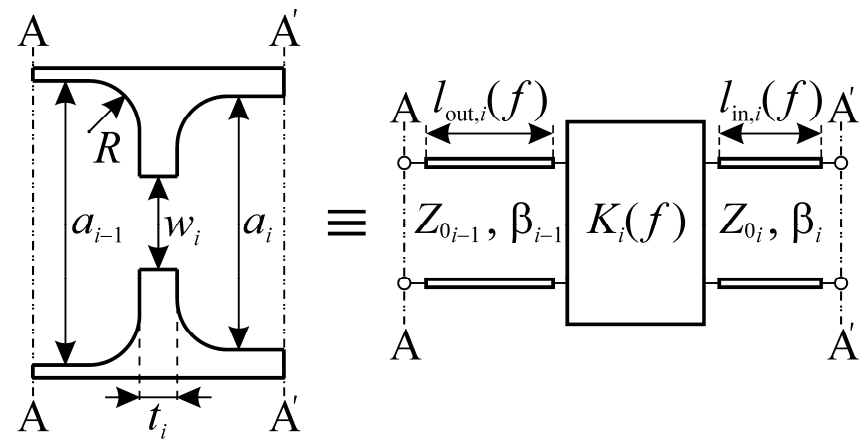

Figure 3. Geometry of the structure $i$-th coupling window and equivalent circuit model obtained from FEST 3D simulations.

\section{B. Electromagnetic Modeling}

The combined prototype-structure synthesis requires EM simulations of the filter coupling windows in two different situations. They are carried out to obtain the window design variables (i.e., lengths $t_{i}$ or widths $w_{i}$ ) providing the parameters $K_{0, i}$ of the prototype impedance inverters at $f_{0, \text { inv }}$. In addition, the coupling windows are also simulated in a wideband to update the prototype parameters $\alpha_{i}, \kappa_{1, i}$ and $\kappa_{2, i}$.

The basic element to be simulated is the real coupling window with rounded corners shown in Fig. 3. This type of coupling window arises when a milling machine is applied to a metallic block. All the rounded corners have the same radius $R$, since the same milling cutter is normally used in the final step of the H-plane filter manufacturing process.

The FEST 3D analysis capabilities allow the accurate and efficient full-wave simulation of arbitrarily shaped $\mathrm{H}$ plane and E-plane components by means of the BI-RME method [18], [19]. A particular element has been created to model 2D generalized inductive coupling windows with rounded corners. The current BI-RME implementation for H-plane components approximates the rounded shapes with linear segments (keeping the waveguide H-plane surface). Since the variations in the filter response due to the rounded corners is limited (as it will be shown in section IV), this approximation is usually valid. Anyway, and following [20], an enhanced module able to rigorously consider circular and elliptic segments is currently under development.

The lengths of the waveguide ports included in the coupling window EM model (see Fig. 3) cause phase shifts in the scattering parameters, which reduce the lengths computed for the waveguide resonators. Obviously, the real lengths of the resonator waveguide sections include the port lengths assigned to the coupling windows in the EM simulations. To reduce the size of the H-plane components and speed up their electromagnetic simulations, short port lengths are chosen.

It is worth stressing that the time required by the BIRME method to compute the response at each frequency point is negligible once the $2 \mathrm{D}$ eigenvalue problem has been solved. Hence, the wideband model of all of the structure coupling windows, required to update the prototype parameters $\alpha_{i}, \kappa_{1, i}$ and $\kappa_{2, i}$, can be computed in very few seconds.

\section{Including the Rounded Corners}

Most of the design methods for H-plane filters with rounded corners reported in the literature start from the design of an equivalent filter with square sharp corners. Since the electric field is close to zero in the emplacement of the rounded corners, suitable initial structure dimensions are normally obtained (i.e., with an error normally in the range of $\pm 100 \mu \mathrm{m}$ for an X-band filter with demanding specifications). From these dimensions, an optimization of the filtering structure is usually carried out. Such optimization involves EM simulations of the whole device with rounded corners, which can be carried out using the finite element method (FEM) [6], the finite difference time domain method (FDTD) [7] or an hybrid modal method [9]. Since an EM simulation of the entire structure is expensive in CPU time and memory terms, the final optimization is usually cumbersome.

The novelty of the design approach proposed in this paper is the inclusion of the rounded corners in a very accurate synthesis procedure. This key point brings two important advantages. Firstly, an excellent structure can be obtained that cannot even require a final EM optimization. Secondly, only isolated coupling windows are simulated instead of the entire structure. These key differences contribute to a drastic reduction in $\mathrm{CPU}$ time, which becomes more important as the filter order increases (and therefore the number of design variables). Additionally, and due to the flexibility and robustness of the synthesis technique [13], the design of almost any practical H-plane filter can be successfully carried out, regardless of their bandwidth, return loss, order or resonators' width choice.

Even under this new approach, it is more advantageous to perform a preliminary synthesis of a conventional H-plane waveguide filter without rounded corners. In fact, any effort devoted to reduce the number of EM simulations of coupling windows with rounded corners improves the synthesis efficiency. The procedure therefore begins with the synthesis of an equivalent filter with sharp corners. The convergence criterion for this preliminary filter is softened by a factor of 4 , as it will only provide the initial dimensions for the filter with rounded corners.

Next, the sharp corners of the conventional H-plane filter just obtained are replaced with rounded corners of radius $R$. Since the frequency response of the new coupling windows will be slightly different, their wideband model is computed to update the prototype parameters $\alpha_{i}, \kappa_{1, i}$ and $\kappa_{2, i}$. A new prototype is thus obtained, which must be synthesized again to recover the ideal response. The physical dimensions of the real coupling windows with rounded corners implementing the synthesized prototype impedance inverters are then extracted. This is clearly the slowest part of the synthesis iteration, as it requires the highest number of EM simulations. A new iteration starts after the wideband 


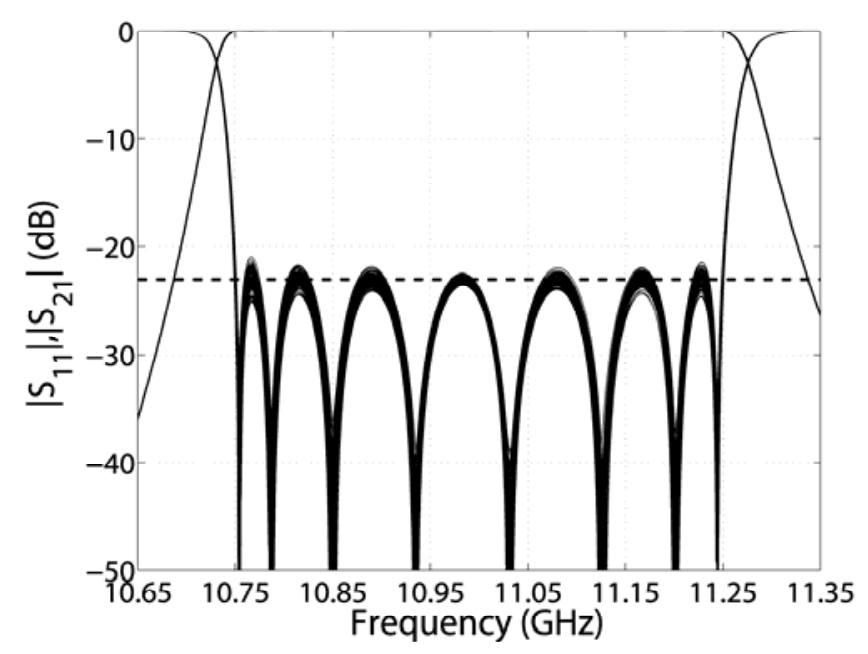

(a)

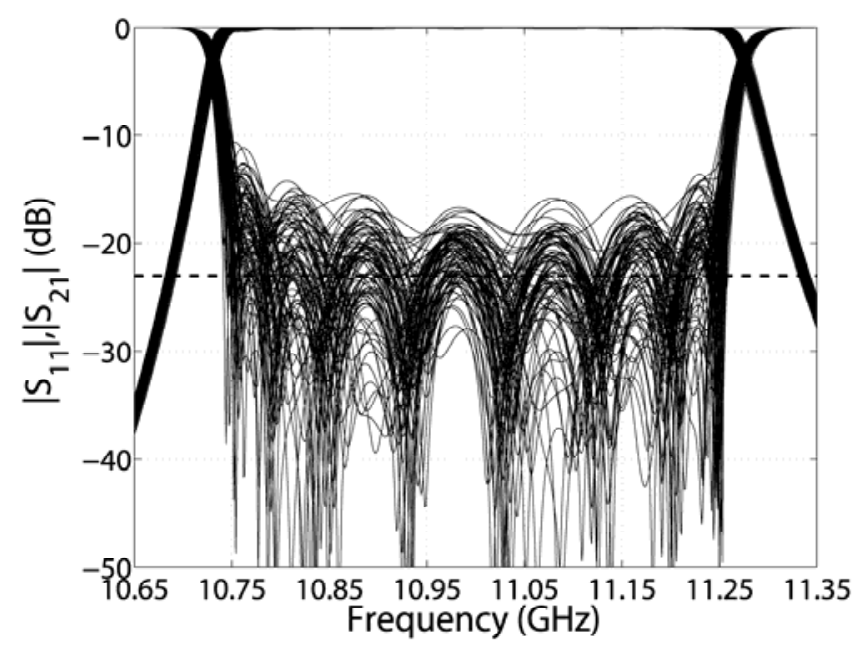

(c)

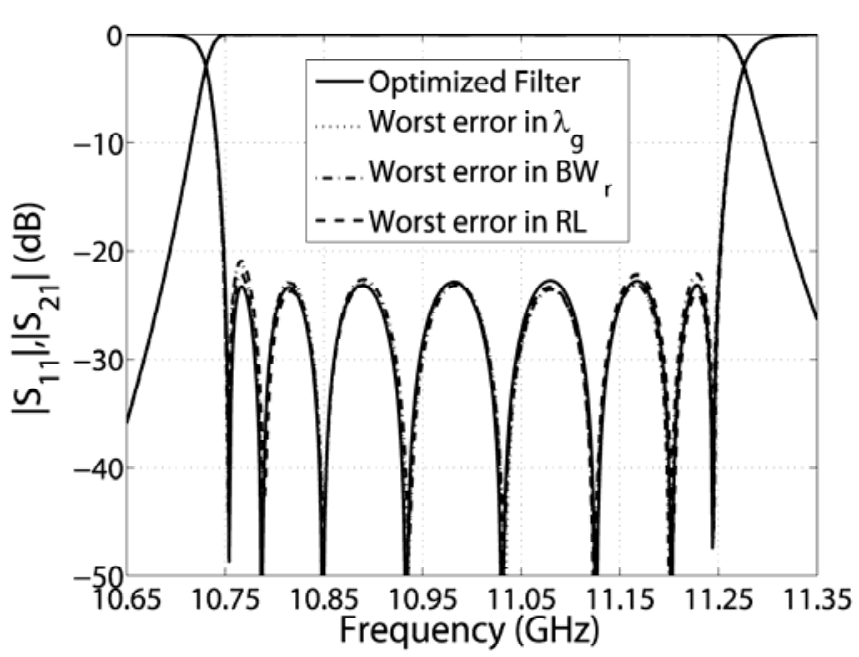

(b)

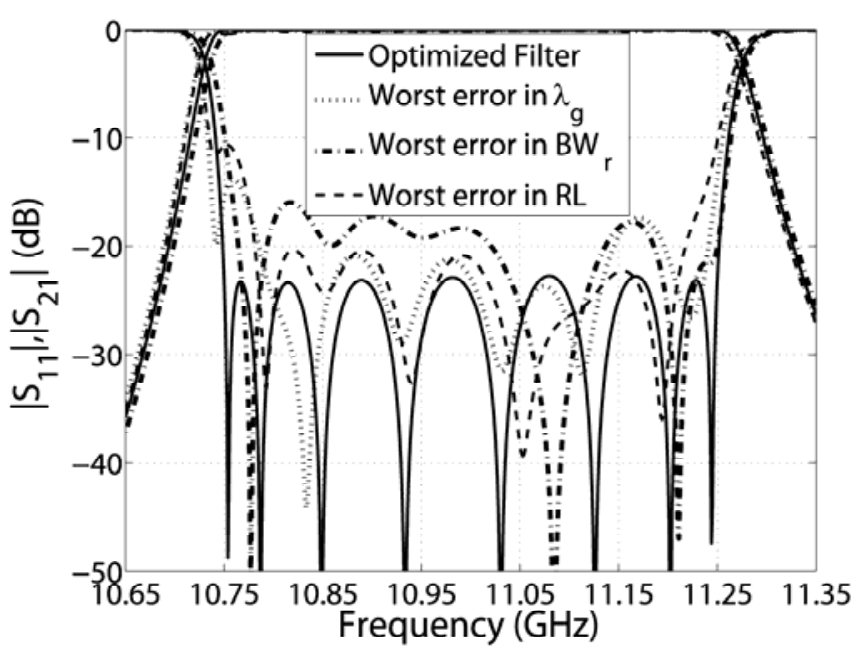

(d)

Figure 4. Simulation in (a) and (c) of the Monte Carlo analyses of a passband filter for $\pm 2.5 \mu \mathrm{m}$ and $\pm 25 \mu \mathrm{m}$ tolerances, respectively. The worst responses for each computed error term are depicted in (b) and (d).

characterization of the new coupling windows, which also update the prototype free parameters. Convergent filter dimensions are normally obtained after one or two additional coupling window extractions.

Using this algorithm, the number of synthesis iterations with rounded corners is reduced. Moreover, the first iteration considers coupling windows with square corners, which can be analyzed in hundredths of a second with FEST 3D pure modal analysis methods [18]. This first synthesis iteration requires a higher number of simulations, since an initial guess of the coupling window dimensions is not available. In fact, the Brent's bracketing algorithm is utilized instead of the faster Newton-Raphson's method used in the remaining iterations.

Once the convergence criterion has been fulfilled, an outstanding structure is usually obtained. In fact, a final EM optimization is not normally required. Anyway, FEST 3D automatically opens an optimization window, thus allowing the user to easily perform a final refinement of the whole structure. To carry out the EM simulation of the entire filter, the generalized admittance matrix of the structure blocks (waveguide uniform sections and coupling windows with rounded corners) are computed. FEST 3D connects these matrices according to the boundary conditions, and solves the resulting linear system to obtain the filter response [18]. The efficiency of this segmented analysis technique is several orders of magnitude better than pure FEM or FDTD approaches, which do not split the structure in smaller parts.

\section{TOLERANCE ANALYSIS}

\section{A. Performance Estimates}

This subsection presents analytical expressions providing error estimates for the response impairments due to a random variation in the filter dimensions. Such expressions must only involve the filter specifications and the manufacturing tolerance, so that a priori estimates can be easily computed. 
To derive the aforementioned expressions, the complete design (i.e., synthesis and final optimization) of a vast range of uniform H-plane filters in the $\mathrm{X}$ band has been carried out. Since the effect of small perturbations of the rounded corners in the filter response is negligible, only square corner filters have been considered. Several Monte Carlo analyses have been performed on each designed filter, using a uniform random variation of the filter physical dimensions. In each analysis, a different maximum value $T$ for the uniform distribution is used (i.e., the manufacturing tolerance). The following relative errors have been measured in the simulated response of each perturbed filter

$$
\begin{gathered}
\operatorname{err}_{\lambda_{\mathrm{g} 0}}=\frac{\left|\lambda_{\mathrm{g} 0}^{(\text {real })}-\lambda_{\mathrm{g} 0}^{\text {(ideal) }}\right|}{\lambda_{\mathrm{g} 0}^{(\text {ideal })}} \\
e r r_{B W_{\mathrm{r}}}=\frac{\left|B W_{\mathrm{r}}^{(\text {real })}-B W_{\mathrm{r}}^{(\text {ideal })}\right|}{B W_{\mathrm{r}}^{(\text {ideal })}} \\
\operatorname{err}_{R L}=\frac{\left|P_{\max }^{-(\text {real })}-P_{\max }^{-(\text {ideal })}\right|}{P_{\max }^{-(\text {ideal })}}=\frac{\left.|| S_{11}\right|_{\text {max }} ^{(\text {real })^{2}}-\left|S_{11}\right|_{\text {max }}^{(\text {ideal })^{2}} \mid}{\left|S_{11}\right|_{\max }^{(\text {ideal })^{2}}}
\end{gathered}
$$

which provide, respectively, the center guide wavelength shift, the relative bandwidth variation and the passband return loss increment (more precisely, the relative increment on reflected power in the poorest matched frequency). To evaluate (3), the starting and ending filter passband frequencies have been measured, as well as the highest reflection coefficient $\left|S_{11}\right|_{\max }^{(\text {real })}$ inside this band. The center guide wavelength $\lambda_{\mathrm{g} 0}$ and relative bandwidth $B W_{\mathrm{r}}$ in both the perturbed (real) and ideal cases are computed by using the procedure in [21].

Figure 4 shows the simulations of two Monte Carlo analyses with different maximum variation $T$ for a filter with $500 \mathrm{MHz}$ bandwidth and $23 \mathrm{~dB}$ return loss ripple, as well as the worst response for each of the three error terms in (3). This figure clearly shows the effect of manufacturing tolerances. Note that the greatest impairment is observed in the passband return loss level, both for slight and severe random perturbations in the filter physical dimensions.

About 30 uniform H-plane filters in X-band have been designed for different sets of specifications, and 16 exhaustive Monte Carlo analyses (with different tolerance T) have been performed on each filter. The three error terms in (3) have been computed for each single perturbed filter, and their root-mean square (RMS) have been fitted to

$$
\operatorname{err}_{\mathrm{x}}(\mathrm{RMS})=\zeta\left(\frac{T}{\lambda_{\mathrm{g} 0}}\right)^{\alpha} \frac{\left(\lambda_{\mathrm{g} 0} / \lambda_{\mathrm{g}, \mathrm{wg}}\right)^{\beta} N^{\varepsilon}}{B W_{\mathrm{r}}^{\chi}\left|S_{11}\right|_{\max }^{\delta}}
$$

where $N$ is the filter order, and $\lambda_{\mathrm{g}, \mathrm{wg}}$ denotes the guide wavelength at 1,575 times the cutoff frequency of the $\mathrm{TE}_{10}$ mode in the resonator waveguides (approximately the center of the waveguide recommended operation band).

Observe that an RMS error term in the form of (4) is dimensionless. In fact, they are expressed in terms of the electrical tolerance $T / \lambda_{\mathrm{g} 0}$ which allows us to extrapolate the results from X-band to any frequency band. Finally, the term $\lambda_{\mathrm{g} 0} / \lambda_{\mathrm{g}, \mathrm{wg}}$ measures the effect of the filter passband position within the operating frequency range of the resonator/port waveguides.

After processing all the results, the final expressions for the root-mean square (RMS) error of the three performances under consideration are

$$
\begin{gathered}
\operatorname{err}_{\lambda_{\mathrm{g} 0}}(\mathrm{RMS})=0.86 T \frac{\lambda_{\mathrm{g} 0}{ }^{0.5} B W_{\mathrm{r}}^{0.1}}{\lambda_{\mathrm{g}, \mathrm{wg}}^{1.5} N^{0.43}} 10^{\frac{0.53}{20} R L(\mathrm{~dB})} \\
\operatorname{err}_{B W_{\mathrm{r}}}(\mathrm{RMS})=1.98 T^{1.04} \frac{\lambda_{\mathrm{g} 0}{ }^{0.23}}{\lambda_{\mathrm{g}, \mathrm{wg}}^{1.27} B W_{\mathrm{r}}^{0.71} N^{0.51}} 10^{\frac{0.63}{20} R L(\mathrm{~dB})} \\
\operatorname{err}_{R L}(\mathrm{RMS})=2.13 T^{1.17} \frac{\lambda_{\mathrm{g} 0}{ }^{0.58} N^{1.82}}{\lambda_{\mathrm{g}, \mathrm{wg}}^{1.75} B W_{\mathrm{r}}^{1.1}} 10^{\frac{1.03}{20}} R L(\mathrm{~dB})
\end{gathered}
$$

where the parameter related to the input reflection coefficient has been expressed in terms of the specified passband return loss $R L$. The maximum error observed is approximately three times the RMS error, and the average error is about $10 \%-20 \%$ below. The RMS error is more conservative than the average error and far less pessimistic than the maximum error, thus being the one we recommend to use.

Using these expressions, a designer can evaluate the expected impairments in the filter performances for a particular manufacturing tolerance. Conversely, they can also be used to find the maximum allowable variation in the filter dimensions required to keep the filter performances within an acceptable range.

Although all the tolerance analyses have been performed for uniform H-plane filters, the results can be extended to inhomogeneous structures with non-equal resonator widths as well. Instead of using the worst width to obtain a conservative estimate, we take the average resonator/port width to compute the parameters $B W_{\mathrm{r}}, \lambda_{\mathrm{g} 0}$ and $\lambda_{\mathrm{g}, \mathrm{wg}}$ in (5). The obtained results also agree well with the simulated performance degradations.

\section{B. Convergence Criterion}

The expressions obtained in subsection III-A can be used to set the synthesis convergence criterion, thus increasing the automation of the CAD procedure. For example, allowing a maximum impairment of $1 \mathrm{~dB}$ in the return loss, from (3c) and (5c) we obtain

$$
S_{1 \mathrm{~dB}}=T=0.17 \frac{B W_{\mathrm{r}}^{0.93} \lambda_{\mathrm{g}, \mathrm{wg}}^{1.49}}{\lambda_{\mathrm{g} 0}{ }^{0.49} N^{1.55}} 10^{-\frac{0.87}{20} R L(\mathrm{~dB})}
$$

so the manufacturing tolerance must be lower than $S_{1 \mathrm{~dB}}$ to keep the return loss variation normally below $1 \mathrm{~dB}$.

Since the return loss always exhibits the most severe degradation among the three error terms computed, the bound (6) could be used to set the synthesis convergence. 
TABLE I. Dimensions of the extracted filters in different iterations of the synthesis procedure and the optimized filter with and without rounded corners

\begin{tabular}{cccccc}
\hline & \multicolumn{3}{c}{ Convergence } & Optimize & $\begin{array}{c}\text { Square } \\
\text { Dimensions }\end{array}$ \\
\cline { 2 - 4 } & $97 \mu \mathrm{m}$ & $10 \mu \mathrm{m}$ & $1.5 \mu \mathrm{m}$ & $\begin{array}{c}\mathrm{d} \\
\text { Filter }\end{array}$ \\
\hline$l_{1}=l_{10}(\mathrm{~mm})$ & 13.314 & 13.409 & 13.400 & 13.396 & 13.332 \\
$l_{2}=l_{9}(\mathrm{~mm})$ & 15.438 & 15.470 & 15.466 & 15.470 & 15.406 \\
$l_{3}=l_{8}(\mathrm{~mm})$ & 15.934 & 15.926 & 15.925 & 15.927 & 15.860 \\
$l_{4}=l_{7}(\mathrm{~mm})$ & 16.044 & 16.035 & 16.035 & 16.036 & 15.969 \\
$l_{5}=l_{6}(\mathrm{~mm})$ & 16.076 & 16.068 & 16.067 & 16.068 & 16.001 \\
\hline$w_{1}=w_{11}(\mathrm{~mm})$ & 11.379 & 11.287 & 11.297 & 11.295 & 11.220 \\
$w_{2}=w_{10}(\mathrm{~mm})$ & 8.056 & 8.026 & 8.031 & 8.024 & 7.992 \\
$w_{3}=w_{9}(\mathrm{~mm})$ & 7.108 & 7.140 & 7.141 & 7.137 & 7.117 \\
$w_{4}=w_{8}(\mathrm{~mm})$ & 6.883 & 6.915 & 6.915 & 6.912 & 6.895 \\
$w_{5}=w_{7}(\mathrm{~mm})$ & 6.812 & 6.842 & 6.842 & 6.840 & 6.822 \\
$w_{6}(\mathrm{~mm})$ & 6.794 & 6.823 & 6.824 & 6.820 & 6.804 \\
\hline
\end{tabular}

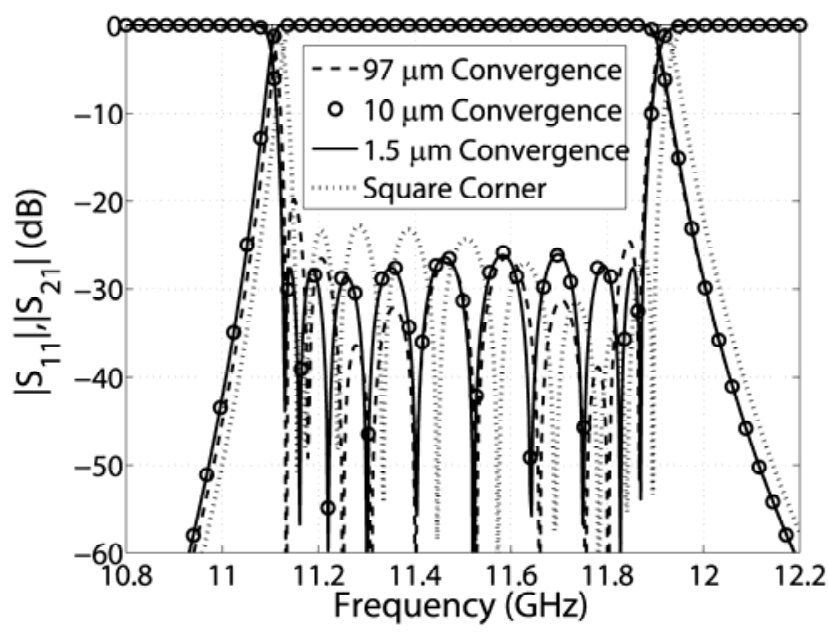

(a)

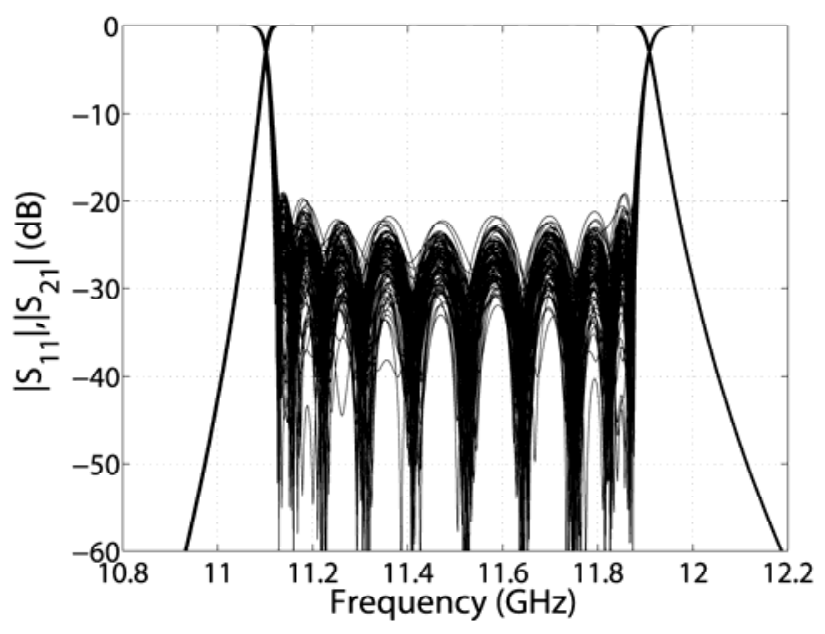

(b)

Figure 5. Response of the extracted filters in different iterations of the synthesis technique in (a). Monte Carlo analysis of the optimized structure with a $\pm 10 \mu \mathrm{m}$ tolerance in (b).

However, and according to our practical experience, this criterion is very restrictive. This fact is because the errors in the physical dimensions of a synthesized filter are not randomly distributed. Contrarily, they are interrelated and tend to compensate. Moreover, the authors have observed that this effect increases with the filter order $N$, as the synthesis errors in the central filter sections are usually very small.

These results suggest that the convergence bound (6) can be relaxed. In fact, it can be replaced without a noticeable variation in the filter response by

$$
S_{\text {ldB }}^{\prime}=\frac{N}{2} S_{\text {IdB }}=0.085 \frac{B W_{\mathrm{r}}^{0.93} \lambda_{\mathrm{g}, \mathrm{gg}}^{1.49}}{\lambda_{\mathrm{g} 0}^{0.49} N^{0.55}} 10^{-\frac{0.87}{20} R L(\mathrm{~dB})}
$$

that although still conservative, is closer to the synthesis sensitivity.

The synthesis method therefore finishes when the maximum difference in the extracted dimensions in two consecutive iterations is lower than $S_{1 \mathrm{~dB}}^{\prime}$. However, this condition implies another extraction of the coupling window dimensions to check the convergence criterion. To avoid this time consuming step, the variation of the structure dimensions with respect to the prototype parameters $\lambda_{\mathrm{g} 0, i}$ and $K_{0, i}$ are stored. After updating the prototype parameters and before carrying out the coupling window extractions, an estimate of the new filter dimensions can be easily computed. Since such estimates are an excellent guess of the real dimensions, they can be used to check the convergence criterion. If the criterion is fulfilled, the synthesis ends without carrying out the last extraction of the coupling windows, thus reducing the synthesis CPU time. Otherwise, they are used as excellent starting points to compute the new window dimensions.

\section{RESULTS}

The fully automated synthesis procedure of H-plane filters with rounded corners just presented has been integrated in the FEST 3D bandpass synthesis tools [17]. All the CPU times in this section have been obtained using 
FEST 3D in a standard laptop PC with a $2 \mathrm{GHz}$ T7250 Intel Core 2 Duo processor.

To test the accuracy of the synthesis method proposed and the tolerance estimates, a 10th order uniform filter in WR75 waveguide and centered at $11.5 \mathrm{GHz}$ has been first considered. The specified passband bandwidth is $750 \mathrm{MHz}$ with a $27 \mathrm{~dB}$ return loss ripple. The radius of the rounded corners has been chosen to be $3.5 \mathrm{~mm}$, and the coupling window thickness has been fixed to $1.5 \mathrm{~mm}$. The synthesis procedure described in this paper took only 65 seconds to extract an excellent filter. If the equivalent square corner filter is not synthesized first, the same structure (differences lower than a micron) is obtained after 91 seconds. A first iteration with rounded corners has been replaced by two faster iterations of a filter with square corners, thus achieving an important time reduction of about $30 \%$.

The dimensions of the extracted structures in the synthesis iterations are compiled in Table I, and their simulated responses are compared in Fig. 5(a). The convergence values denote the expected maximum variation in the filter dimensions for the next structure extraction (whose error with respect to the real variation is usually lower than $2 \%$ ). The filter with $97 \mu \mathrm{m}$ convergence is unacceptable, whereas the responses of the $10 \mu \mathrm{m}$ and 1.5 $\mu \mathrm{m}$ convergence filters are very similar. According to (6), the computed filter sensitivity $S_{1 \mathrm{~dB}}$ for a $1 \mathrm{~dB}$ return loss variation is about $1.5 \mu \mathrm{m}$, whereas the adjusted sensitivity $S_{1 \mathrm{~dB}}^{\prime}$ in (7) set the convergence criterion to a more realistic value of $\pm 7 \mu \mathrm{m}$. The iteration providing the $1.5 \mu \mathrm{m}$ convergence filter was not performed, since the expected variations in the filter physical dimensions are below the convergence criterion. Anyway, such estimated variations are added to the final structure dimensions to increase the synthesis accuracy.

The resulting filter shows a degradation of barely \pm 1.2 $\mathrm{dB}$ in the return loss ripple, and a passband shift of only 100 $\mathrm{KHz}$. A final EM optimization is not usually required to correct these small impairments, which are below typical manufacturing tolerances and are close to the accuracy of EM simulators. The synthesis method has clearly reached convergent results, as the response differences between 10 $\mu \mathrm{m}$ and $1.5 \mu \mathrm{m}$ filters are negligible. The small degradations can be attributed to the slight inaccuracies of the synthesis method; mainly due to the higher order modes neglected in the prototype model (see Fig. 2). These results also reveal that $S_{1 \mathrm{~dB}}^{\prime}$ provides a good and somewhat conservative synthesis convergence bound.

A viability study on a manufacturing procedure with a tolerance of $\pm 10 \mu \mathrm{m}$ has been carried out. The expected impairments have been evaluated by means of (5), and verified with the Monte Carlo analysis whose simulations are shown in Fig. 5(b). The estimated and measured RMS errors in the center wavelength are only $0.048 \%$ and $0.046 \%$, respectively (i.e., about $3 \mathrm{MHz}$ of frequency shift). The passband bandwidth RMS variation in both cases is
TABLE II. Dimensions of the $7^{\text {th }}$ order non-uniform filter

\begin{tabular}{cccccc}
\hline & \multicolumn{2}{c}{ Coupling Windows } & & \multicolumn{2}{c}{ Resonators } \\
\cline { 2 - 3 } \cline { 5 - 6 }$i$ & $w_{i}(\mathrm{~mm})$ & $t_{i}(\mathrm{~mm})$ & & $a_{i}(\mathrm{~mm})$ & $l_{i}(\mathrm{~mm})$ \\
\hline 1 & 16.748 & 2.500 & & 28.500 & 23.897 \\
2 & 11.072 & 2.500 & & 31.000 & 25.113 \\
3 & 9.599 & 2.500 & & 34.240 & 24.051 \\
4 & 9.222 & 2.500 & & 35.600 & 23.673 \\
5 & 9.183 & 2.500 & & 36.120 & 23.475 \\
6 & 9.385 & 2.500 & & 36.320 & 23.088 \\
7 & 10.502 & 2.500 & & 36.500 & 20.614 \\
8 & 16.247 & 2.500 & & - & - \\
\hline
\end{tabular}

$0.46 \%$, namely, lower than $4 \mathrm{MHz}$. As expected, the return loss exhibits the more important degradation. Equation (5c) estimates an error of 2.56, which translates to $5.5 \mathrm{~dB}$, whereas the measured RMS variation is about $6.1 \mathrm{~dB}$. These results demonstrate the accuracy of (5) to estimate the expected variation in the filter performance. From these data, the designer can determine whether to accept or not a manufacturing procedure with $\pm 10 \mu \mathrm{m}$ tolerance. A comparison between figures 5(a) and 5(b) clearly shows that random variations of $\pm 10 \mu \mathrm{m}$ in the filter dimensions produces a substantially higher degradation than a maximum error of $\pm 10 \mu \mathrm{m}$ due to the synthesis procedure accuracy.

In order to evaluate the effect of the rounded corners, the filter has also been synthesized and optimized without considering its $3.5 \mathrm{~mm}$ radius rounded corners. In spite of this approximation, the difference in the filter dimensions are only in the range between 15 and $75 \mu \mathrm{m}$ (see Table I). Figure 5(a) shows a $30 \mathrm{MHz}$ passband frequency shift as well as moderate return loss impairment in the simulated response of this filter (including its rounded corners). Rounded corners with radii lower than $1.5 \mathrm{~mm}$ in X-band filters scarcely degrade the structure response, and can even be neglected.

For the last example a 7 th order C-band filter centered at 7.25 GHz with $5 \mathrm{~mm}$ radius rounded corners was synthesized. The filter bandwidth was $300 \mathrm{MHz}$ and the passband return loss was set to $23 \mathrm{~dB}$. The filter ports were standard WR137 waveguides. A rejection greater than $70 \mathrm{~dB}$ from 14.1 to $15 \mathrm{GHz}$ (including the second harmonic frequency band) was specified. This stopband requirement can only be met with an inhomogeneous filter with nonequal resonators. The widths of the filter resonators were chosen to provide some transmission zeros in the 2nd harmonic frequency band through the negative coupling between the propagating $\mathrm{TE}_{10}$ and $\mathrm{TE}_{30}$ modes [22]. The synthesized filter topology and its passband and wideband responses are shown in Fig. 6. The extracted filter dimensions are compiled in Table II.

The filter stopband fulfills the demanding specifications (see Fig. 6(b)), with a rejection greater than $80 \mathrm{~dB}$ in the specified range including the second harmonic frequencies. The position of the transmission zeros provides an almost equiripple attenuation in such a frequency range. 


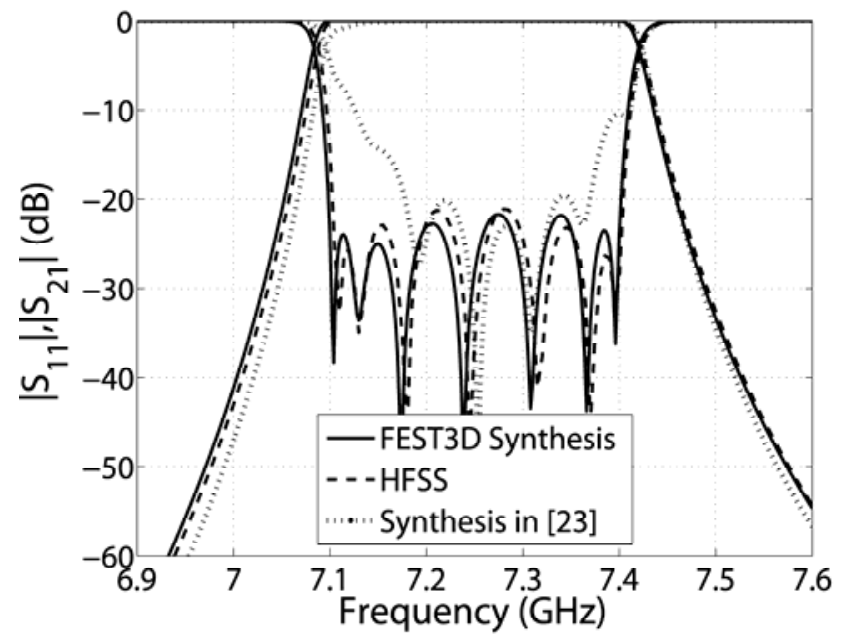

(a)

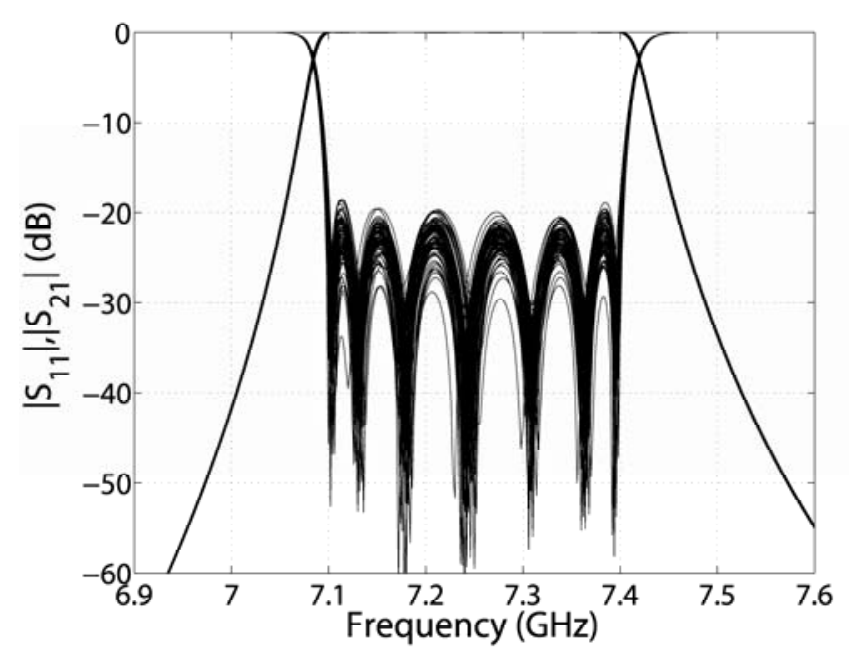

(c)

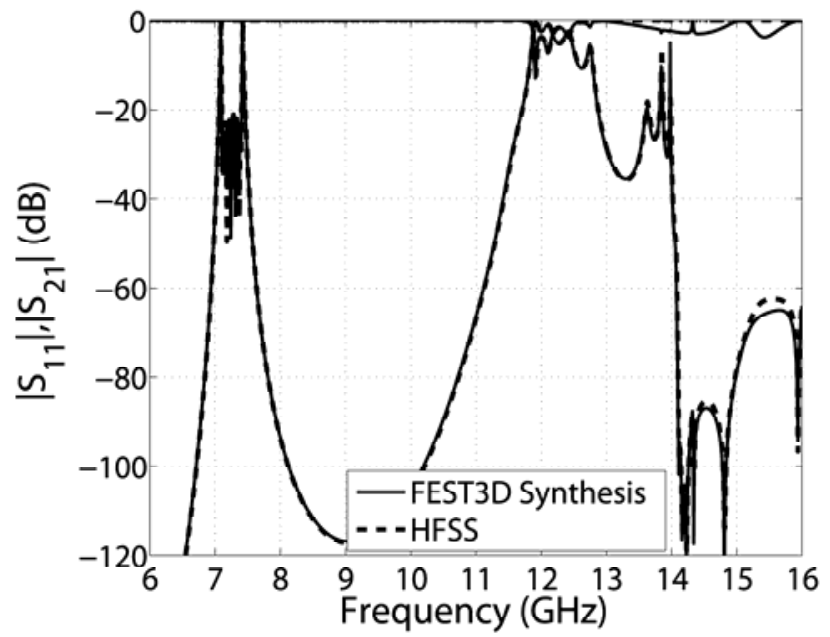

(b)

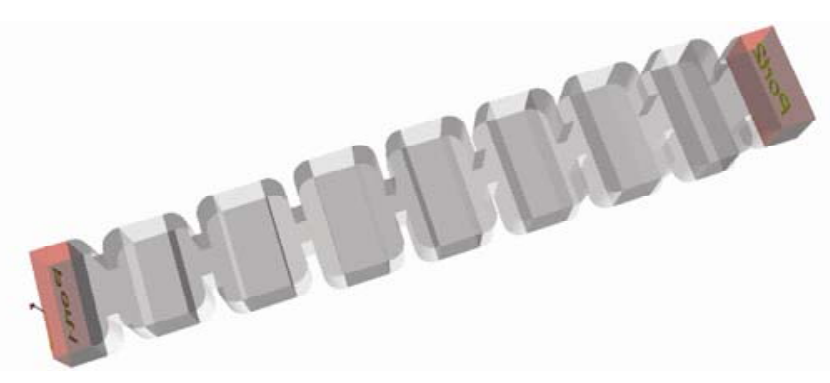

(d)

Figure 6. Simulated passband (in (a)) and wideband (in (b)) responses of the synthesized filter with rounded corners obtained from FEST3D and HFSS, and comparison with the response of the extracted structure obtained by using the technique in [23]. A Monte Carlo analysis for a maximum variation of $\pm 10 \mu \mathrm{m}$ is shown in (c). The filter synthesized by FEST 3D is depicted in (d).

The passband response depicted in Fig. 6(a) is also very good. The variations in the passband ripple, always below $22 \mathrm{~dB}$, are due to the higher order modes in the wider resonators. These modes are neglected in the prototype and the synthesis procedure, and introduce small variations in the filter response. If required, the exact equiripple response can be recovered with a slight refinement. This final optimization can be easily performed in FEST 3D due to the excellent initial dimensions and the efficiency of its fullwave analysis methods (partly described in section II-B). For comparative purposes, the response of the extracted structure with the method in [23] is also shown. Although this method is a generalized version of the classic technique in [24] for the inhomogeneous case, it neglects the diverse frequency dependence of the different structure parts. As a result, its extracted filter response is only good close to the passband center frequency, and the average error in the filter dimensions is about $250 \mu \mathrm{m}$. A cumbersome optimization from these poor initial dimensions must be performed. The design of inhomogeneous structures by traditional techniques is a hard task. Using the approach in this paper, however, they can be accurately synthesized even including the rounded corners arising from manufacturing.

The passband and stopband HFSS responses in Fig. 6 show an upwards frequency shift of about $4 \mathrm{MHz}$, which can be attributed to the HFSS linear segmentation of rounded contours [25]. HFSS replaces circular contours with circumscribed polygons. Since this replacement increases the metallic area, the H-plane surface of the waveguide resonators is slightly reduced. As a result, the filter response is moved upwards in frequency. This very small and similar geometrical change in all the filter resonators explains the slight frequency shift of about $0.06 \%$, and also the nearly unaltered return loss ripple of the HFSS response when compared with FEST3D. In fact, an increase in the number of linear segments tends to move the HFSS response downwards in frequency. 
The convergence bound (7) is about $\pm 13.5 \mu \mathrm{m}$ for the average resonator/port width. The synthesis of this structure is performed with two iterations without and with rounded corners, and takes only 67 seconds. If the synthesis is performed directly, four iterations with rounded corners are required to obtain convergent results after 169 seconds. It is worth saying that asymmetric filters are more demanding than symmetric ones, as the number of coupling windows to be synthesized is doubled.

Provided that the maximum tolerable reduction in return loss is $3 \mathrm{~dB}$, the expressions (3c) and (5c) suggest a manufacturing tolerance below $\pm 11 \mu \mathrm{m}$. A Monte Carlo tolerance analysis with a maximum perturbation of $\pm 10 \mu \mathrm{m}$ is shown in Fig. 6(c). Since the estimates provide RMS variation instead of maximum variation, the return loss can be slightly lower than $20 \mathrm{~dB}$ in some unfortunate cases. If a maximum variation of $3 \mathrm{~dB}$ in the passband return loss must be guaranteed, a more exacting bound should be taken. In fact, using a \pm 5 microns tolerance, the filter can always be implemented without tuning elements obtaining a return loss greater than $20 \mathrm{~dB}$ in the passband.

The results of the Monte Carlo analysis in Fig. 6(c) agree well with the values provided by the error estimators (5), and the tolerance and convergence figures obtained in this particular example (for instance, the difference between the expected and the simulated RMS variation in the ripple level is only $0,15 \mathrm{~dB}$ ). These results prove the applicability of the analytical expressions derived in this paper to any frequency range, and their suitability for inhomogeneous structures as well.

\section{CONCLUSIONS}

This paper is focused on the modeling of manufacturing defects in waveguide filters. More precisely, it has dealt with the rounded corners due to milling machining processes and the random variations in the structure physical dimensions related to manufacturing tolerances.

An accurate design procedure able to take into account the rounded corners has been proposed. In contrast to other methods proposed in the technical literature, the rounded corners have been included during the synthesis stage. Advantageously, a very efficient and robust method has been obtained which provides outstanding initial filter dimensions that, at worst, only need a slight refinement. On the other hand, a statistical characterization has been carried out to evaluate the impact of the random variations of the filter dimensions due to manufacturing tolerances. As a result, analytical estimators for the deterioration of the main filter performances have been derived. These types of expressions are of interest to designers, since they can be used to determine the manufacturing tolerance to be requested for a tuning-less filter implementation. Moreover, modified versions of these estimators can also be used to set the convergence criteria in fully automated CAD procedure.
Using the design technique and the estimates described in this paper, practitioners can easily consider the two most important manufacturing effects during the filter design. Since they can be of help to reduce component development times and costs, both enhancements have been successfully integrated in FEST 3D.

\section{ACKNOWLEDGEMENT}

The authors would like to thank Mrs. Eva Tarín, Mr. Óscar Monerris and Mr. Jaime Armendariz for their work on the integration of the synthesis tool for H-plane waveguide filters in FEST 3D. This work was supported by Ministerio de Educación y Ciencia, Spanish Government, under the Research Project ref. TEC2010-21520-C04-01.

\section{REFERENCES}

1. J. M. Reiter and F. Arndt, Rigorous analysis of arbitrarily shaped $\mathrm{H}$ - and E-plane discontinuities in rectangular waveguide by a full-wave boundary contour mode-matching method, IEEE Trans Microwave Theory Tech 43 (1995), 796-801.

2. J. Zhou, B. Duan, and J. Huang, Influence and tuning of tunable screws for microwave filters using least squares support vector regression, Int J RF and Microwave CAE 20 (2010), 422-429.

3. J. W. Bandler, Ed., IEEE Trans Microwave Theory Tech, Special Issue on Automated Circuit Design Using Electromagnetic Simulators 45 (1997), no. 5.

4. IEEE Microwave Magazine, Special Issue on Electromagnetic Software, 9 (2008), no. 6.

5. V. E. Boria, B. Gimeno, S. Marini, M. Taroncher, S. Cogollos, P. Soto, A. Vidal, and J. Gil, Recent advances in modeling, design and fabrication of microwave filters for space applications, Int J RF and Microwave CAE 17 (2007), 70-76.

6 J. W. Bandler, R. M. Biernacki, S. H. Chen, and D. Omeragic, Space mapping optimization of waveguide filters using finite element and mode-matching electromagnetic simulators, IEEE MTT-Symp Dig, Denver, CO, June 1997, pp. 635-638.

7. P. Kozakowski and M. Mrozowski, Gradient-based optimization of filters using FDTD software, IEEE Microwave Wireless Compon Lett 12 (2002), 389-391.

8. V. E. Boria, M. Bozzi, F. Bruni, S. Cogollos, G. Conciauro, B. Gimeno, and L. Perregrini, Efficient analysis of in-line waveguide filters and frequency selective surfaces with stepped holes, Int J RF and Microwave CAE 13 (2003), 306-315.

9. J. V. Morro, H. Esteban, V. E. Boria, C. Bachiller, and A. Belenguer, Optimization techniques for the efficient design of low-cost satellite filters considering new light materials, Int J RF and Microwave CAE 18 (2008), 168-175.

10. G. L. Matthaei, L. Young, and E. M. T. Jones, Microwave Filters, Impedance-Matching Networks, and Coupling Structures, Artech House, Norwood, MA, 1980.

11. I. C. Hunter, Theory and Design of Microwave Filters, IET Press, London, UK, 2001.

12. P. Soto and V. E. Boria, A versatile prototype for the accurate design of homogeneous and inhomogeneous wide bandwidth directcoupled-cavity filters, in IEEE MTT-Symp Dig, Fort Worth, TX, June 2004, pp. 451-454.

13. P. Soto, E. Tarín, V. E. Boria, C. Vicente, J. Gil, and B. Gimeno, Accurate synthesis and design of wideband and inhomogeneous inductive waveguide filters, IEEE Trans Microwave Theory Tech 58 (2010), 2220-2230.

14. A. Morini, G. Venanzoni, and T. Rozzi, A new adaptive prototype for the design of side-coupled coaxial filters with close correspondence to the physical structure, IEEE Trans Microwave Theory Tech 54 (2006), 1146-1153. 
15. A. Morini, G. Venanzoni, M. Farina, and T. Rozzi, Modified adaptive prototype inclusive of the external couplings for the design of coaxial filters, IEEE Trans Microwave Theory Tech 55 (2007), 1905-1911.

16. M. Guglielmi and G. Connor, Industrial implementation of tuning-less microwave filters, Microwave Engineering Europe, no. 1 , pp. 39-40, 1996.

17. FEST 3D 6.5, Aurora Software and Testing S.L., on behalf of ESA/ESTEC, Valencia, Spain, 2010, http://www.fest3d.com.

18. G. Conciauro, M. Guglielmi, and R. Sorrentino, Advanced Modal Analysis. John Wiley \& Sons, Chichester, UK, 2000.

19 P. Arcioni, M. Bressan, G. Conciauro, and L. Perregrini, Generalized Y-matrix of arbitrary H-plane waveguide junctions by the BI-RME method, in IEEE MTT-Symp Dig, Denver, CO, June 1997, pp. 211-214.

20. S. Cogollos, S. Marini, V. E. Boria, P. Soto, A. Vidal, H. Esteban, J. V. Morro, and B. Gimeno, Efficient modal analysis of arbitrarily shaped waveguide components composed of linear, circular, and elliptical arcs using the BI-RME method, IEEE Trans Microwave Theory Tech 51 (2003), 2378-2390.

21. R. Levy, Theory of direct-coupled-cavity filters, IEEE Trans. Microwave Theory Tech, 15 (1967), 340-348.

22. M. Guglielmi, P. Bertalan, and A. Nguyen, Accurate CAD of integrated bandpass and second harmonic band-reject microwave filters, in IEEE MTT-Symp Dig, Baltimore, MD, June 1998, pp. 813-816.

23. R. Balasubramanian and P. Pramanick, Computer-aided design of H-plane tapered corrugated waveguide bandpass filters, Int J RF and Microwave CAE, 9 (1999), 14-21.

24. J. D. Rhodes, Theory of Electrical Filters. John Wiley \& Sons, New York, NY, 1976.

25. HFSS Release 13.0, Ansys Inc., Canonsburg, PA, 2011. http://www.ansys.com 